As this tendency develops, it might open up careers for those taking a diploma in social science or pursuing a short course of social research, provided they possessed tho essential qualities of leadership.

Here are suggestions which should be welcome to many scientific workers who are turning their attention to social problems. For some of them the voluntary work which will long be required may offer an opportunity for the expression of their sense of social responsibility, and for maintaining or even raising the standard of leadership. Others may find a like opportunity in the social surveys and investigations which are still necessary to render further information available as a basis for the national action required. For all, however, there is the opportunity to assist by pressing for adequate attention to the problem by local and national educational authorities, by supporting with their active interest such work as is already being carried out in this field by the universities, by bringing sympathy and insight and creative thought to bear on the problems which the needs of youth present to-day. Nor should scientific workers be unmindful of their responsibility as citizens to protest against any hesitation by public authorities to exercise their powers of compulsion to stop certain abuses which at present, as Dr. Morgan so clearly shows, are detrimental to both the health and morale of youth, and, in effect, squander capital which grows the more precious in a time of emergency.
R. Brightrman.

\title{
PLANETS AND SUNSPOTS
}

Tidal Forces in the Sun's Corona due to Planets By K. G. Meldahl. Pp. $14+7$ plates. (Copenhagen: Berlingske Forlag, 1938.) n.p.

THE author of this work believes that radiation pressure on certain small particles in the corona should balance gravitation so closely that a 'sensitive layer' exists. If this is disturbed, it will undergo violent motion and probably ejection. The force likely to produce the disturbances is the tidal action due to the planets, and though this is too weak to cause any noticeable motion in the heavier, luminous portion of the sun's gases, nevertheless, as the hydrogen prominences appear at a level comparable in height to the altitude of the 'sensitive layer', it is important to seek for some connexion between the disturbances and tidal forces. Only five planets need be considered to contribute anything of importance to the tidal force-Mercury, Venus, Earth, Jupiter and Saturn. Mercury, though very small, must we taken into account because the tidal force varies inversely as the cube of the distance of the planet from the sun; Mars is neglected as its tidal force is only about 3 per cent that of the earth. The five tidal forces are added vectorially, the resultant vector giving the height $h$ of the force wave running round the equator, and the main investigation is carried out on these lines.

The period of Mercury is close to 88 days, and for this reason diagrams of vectorial additions for every eleventh day are drawn from 1663 to 1966 to test for periodicities. It does not appear that the period of Jupiter or the longer secular period of the three inner planets taken together is concerned, but a period of 243 years is partly noticeable, and a multiple of this seems to be indicated.
Discussing the connexion between the diagrams and the observations of disturbances, the author directs attention to two important matters. (1) Where radiation pressure is not constant, an increase in it would drive out the previously balanced particles and bring heavier ones into floating equilibrium, thus facilitating disturbances in the lower layers of the corona. (2) If existing spots increase radiation locally, this provides the possibility for relatively smaller tidal forces to generate disturbances. It will be misleading to compare the periodicity of observed spots and group sizes with tidal forces; the latter should be compared with the genesis of spots, and the existing spots should generally show a lag.

The mechanism of spot creation is considered to be as follows: Gases of the sensitive layer rotating with the sun at a speed of about $2 \mathrm{~km}$./sec. run through the tidal field of force. At times they encounter forces greater than those that permit quiescent motion, and there ensues a break-up into vortices such as hydrogen prominences. A detailed description is given for the appearances of spots with regard to certain physical features, but it is open to some doubt whether all these are fully explained by the supposed mechanism of spot creation.

The author predicted a number of disturbances recently, which have since been observed, and it is possible that he has opened the door for further investigation into the genesis of sunspots, auroras, magnetic storms, etc. If it is true, as he states, that prominences are started in the corona by external forces and that spots are the consequence, a fruitful field for research lies in this realm.

HI. D. 